\title{
Barreras y facilitadores del sistema de salud relacionadas con el seguimiento de anormalidades citológicas, Medellín-Colombia*
}

\section{Barriers and Facilitators of the Health System Related to the Follow-Up of Cytological Abnormalities, Medellin-Colombia}

\section{Barreiras e facilitadores do sistema de saúde relacionadas com o monitoramento de anormalidades citológicas, Medellin-Colômbia}

Fecha de recepción: 18-03-14 Fecha de aceptación: 23-09-14 Disponible en línea: 01-07-14

doi: 10.11144/Javeriana.rgyps13-27.bfss

Cómo citar este artículo:

Garcés-Palacio IC, Rubio-León DC, Ramos-Jaraba SM. Barreras y facilitadores del sistema de salud relacionadas con el seguimiento de anormalidades citológicas, Medellín-Colombia. Rev. Gerenc. Polít. Salud. 2014; 13(27): 200-211. http://dx.doi.org/10.11144/Javeriana.rgyps13-27.bfss

\author{
Isabel Cristina Garcés-Palacio** \\ Diana Carolina Rubio-León*** \\ Sara Milena Ramos-Jaraba****
}

Artículo de investigación derivado del proyecto Creencias en salud asociadas con el acceso al diagnóstico y tratamiento de anormalidades citológicas: Perspectiva de las mujeres del régimen subsidiado de la ciudad de Medellín, financiado por el Fondo de Apoyo a la Investigación Docente de la Facultad de Salud Pública de la Universidad de Antioquia. Fecha de inicio: 10 de junio del 2011; fecha de finalización: 30 de noviembre del 2012. Instituciones involucradas: ESE Metrosalud y Universidad de Antioquia. Dirigir correspondencia a: Isabel Cristina Garcés Palacio Calle 62 No.52-59 Oficina 202 Teléfono: 219 6833. Correo electrónico: isabelgarcesp@gmail.com

** Dr.PH y magíster en Salud Pública, profesora asistente de la Facultad Nacional de Salud Pública, Grupo de Epidemiología de la Universidad de Antioquia, Medellín, Colombia. Correo electrónico: isabelgarcesp@gmail.com

**** Magíster en Psicología y Salud, Facultad Nacional de Salud Pública, Universidad de Antioquia, Medellín, Colombia. Correo electrónico: dcrubiol@unal.edu.co

***** Administradora en Salud con énfasis en gestión de servicios de salud, joven investigador de Colciencias, Facultad Nacional de Salud Pública, Universidad de Antioquia, Medellín, Colombia. Correo electrónico: sararamosj@gmail.com 


\section{Resumen}

Objetivos: identificar barreras y facilitadores del seguimiento: diagnóstico y tratamiento de anormalidades citológicas en mujeres de bajos ingresos usuarias de la red pública de servicios de salud de la ciudad de Medellín, Colombia. Métodos: se realizaron ocho grupos focales (62 mujeres) según grupos de edad (25-45 y 46-69 años) y se hizo un análisis de contenido. Resultados: las barreras del sistema de salud reportadas fueron: a) barreras estructurales: fragmentación en la prestación del servicio, problemas con afiliaciones y características del régimen de afiliación; b) barreras administrativas: problemas y demoras en la asignación de citas, largas filas, problemas con autorizaciones y maltrato; y c) barreras económicas derivadas de las dos anteriores y relacionadas con el gasto de bolsillo. También se mencionaron facilitadores estructurales y administrativos. Conclusión: algunas características estructurales del sistema de salud y de la administración de las aseguradoras limitan el acceso de las mujeres al seguimiento de lesiones precancerosas.

Palabras clave: accesibilidad; oportunidad; seguimiento; cáncer de cuello uterino; barreras; anormalidades citológicas

\section{Abstract}

Objectives: Identifying barriers and facilitators of the diagnostic monitoring and treatment of cytological abnormalities in low-income women users of the public health service network in Medellin, Colombia. Methods: We created eight focal groups (62 women) according to age groups (ages 25-45 and 46-69), and performed a content analysis. Results: The reported barriers of the health system were: a) structural barriers: fragmentation in the provision of the service, problems with the affiliations and characteristics of the affiliation regime; b) management barriers: problems and delays in the assignment of appointments, long queues, problems with authorizations and mistreatment; and c) economic barriers derived from the aforementioned and related with expenses. Management and structural facilitators were also mentioned. Conclusion: Some structural characteristics of the health system and the management of insurance companies limit access for women to the monitoring of pre-cancerous lesions.

Keywords: accessibility; opportunity; monitoring; cervical cancer; barriers; cytological abnormalities

\section{Resumo}

Objetivos: identificar barreiras e facilitadores do seguimento: diagnóstico e tratamento de anormalidades citológicas em mulheres de baixa renda usuárias da rede pública de serviços de saúde da cidade de Medellín, Colômbia. Métodos: Realizaram-se oito grupos focais (62 mulheres) de acordo com as faixas etárias (25-45 e 46-69 anos) e se fez análise de conteúdo. Resultados: as barreiras do sistema de saúde reportadas foram: a) barreiras estruturais: fragmentação na prestação do serviço, problemas com afiliações e caraterísticas do régime de afiliação; b) barreiras administrativas: problemas e atrasos na alocação de citas, longas filas, problemas com autorizações e abuso; e c) barreiras económicas decorrentes das duas anteriores e relacionadas com a caixa pequena. Mesmo foram mencionados facilitadores estructuráis e administrativos. Conclusão: algumas caraterísticas estruturais do sistema de saúde e da administração das asseguradoras limitam o acesso das mulheres ao acompanhamento de lesões pré-cancerosas.

Palavras-chave: acessibilidade; oportunidade; acompanhamento; câncer de colo uterino; barreiras; alterações citológicas 


\section{Introducción}

El cáncer de cuello uterino $(\mathrm{CaCu})$ es una enfermedad cuya distribución global es inequitativa, por lo que se le puede considerar una patología innecesaria, evitable e injusta (1). Esta enfermedad afecta principalmente a las mujeres de países de bajos ingresos $(2,3)$, quienes representan el $88 \%$ de las muertes (4). En Colombia, se calcula una incidencia anual de 21,5 casos y una mortalidad de 10 mujeres por cada 100000 (5).

Por lo general, la prevención del $\mathrm{CaCu}$ se centra en el tamizaje con citología vaginal para la detección temprana de lesiones precancerosas; (6) sin embargo, es el seguimiento, referido al diagnóstico completo y al tratamiento de la anormalidad, la estrategia que reduce la mortalidad por $\mathrm{CaCu}$ de forma más efectiva (7-9).

Mientras en países de altos ingresos se ha disminuido la enfermedad al implementar programas que incluyen educación, ejecución de sistemas de información, captación de mujeres, entrega oportuna de resultados, confirmación mediante colposcopia-biopsia y tratamiento oportuno, en los países de bajos ingresos, con recursos e infraestructura insuficientes, se observa seguimiento escaso de las anormalidades, múltiples visitas a los centros de salud y falta de acceso oportuno a la atención médica (6).

En América Latina la estrategia de prevención del $\mathrm{CaCu}$ se ha centrado en el aumento de la cobertura de la citología vaginal, descuidando el control de calidad de la prueba y el seguimiento de las mujeres con reporte de anormalidad (7). En el caso de Colombia, aunque el Sistema General de Seguridad Social en Salud (sGSss) reglamenta que todas las mujeres afiliadas con edades entre 25 y 69 años, así como las menores de 25 años que tengan relaciones sexuales, tienen todas el derecho a la realización de la citología, sin costo (5), y que la citología y la colposcopia-biopsia estén incluidas en el Plan Obligatorio de Salud (POs) para ambos regímenes del sistema: contributivo - personas con vinculación laboral y capacidad de pago - y subsidiado — personas identificadas como pobres por el Sistema de Identificación de Beneficiarios (Sisben), que no tienen capacidad de pago, y pobres no aseguradas: identificadas como pobres por el Sisben, no afiliadas al régimen contributivo, a un régimen excepcional o al régimen subsidiado- Se observa una disociación entre las actividades de tamización, diagnóstico y tratamiento, ya que este último no es de obligatorio cumplimiento para las aseguradoras que atienden a las mujeres de escasos recursos (10).

La más reciente encuesta nacional de salud mostró que $90 \%$ de las mujeres entre 18 y 69 años se habían tomado la citología alguna vez en la vida, mientras que sólo el $61 \%$ dijo que se la tomaba anualmente (5). La cobertura de la citología vaginal es solo una parte de la prevención secundaria del $\mathrm{CaCu}$, por ello la falta de un programa integral que incluya el seguimiento de la anormalidad limita los alcances de las estrategias implementadas $(9,11)$. Se reporta que en Colombia más de un cuarto de las mujeres con anormalidad citológica no acceden al seguimiento requerido $(10,12)$.

En el departamento de Antioquia se observan diferencias en la mortalidad por $\mathrm{CaCu}$ a lo largo de las subregiones, según su nivel de desarrollo socioeconómico. Mientras en la capital, Medellín, se ha observado una tendencia a la disminución de las muertes asociadas a la enfermedad, en las subregiones donde hay mayor pobreza se ha reportado una mortalidad tres veces mayor (13). La relación entre la mortalidad por $\mathrm{CaCu}$ y el nivel de pobreza sugiere problemas en 
el acceso y la oportunidad del tamizaje y el seguimiento de las anormalidades citológicas por parte de las mujeres de escasos recursos.

Se afirma que la calidad de la atención en salud implica garantizar la accesibilidad y la oportunidad en la atención (14). La accesibilidad es la posibilidad que tiene el usuario de utilizar los servicios de salud, según las condiciones: geográfica, administrativa, económica, cultural o simbólica (15); y también según las condiciones financieras y propias de las institución de salud (16). La oportunidad se refiere a la posibilidad que tiene el usuario de obtener los servicios que requiere sin que se presenten retrasos que pongan en riesgo su vida o su salud; es evaluada según el tiempo necesario para obtener una cita y el tiempo de espera en los servicios de salud antes de recibir atención (17). La oportunidad de la atención se relaciona con la organización de la oferta de servicios según la demanda y con la coordinación institucional para gestionar el acceso (14).

Según lo anteriormente expuesto, se consideró pertinente indagar por las barreras y los facilitadores del sistema de salud que se relacionan con la accesibilidad y la oportunidad del seguimiento de las anormalidades citológicas, según la experiencia de un grupo de mujeres de bajos ingresos que asistieron a la red pública hospitalaria de la ciudad de Medellín, Colombia, durante el 2011.

\section{Métodos}

\section{Diseño}

Estudio cualitativo de tipo fenomenológico.

\section{Participantes}

Las mujeres fueron contactadas a partir de listados de cuatro Unidades Intermedias de atención en salud (UI) de la red pública hospi- talaria de la ciudad de Medellín. A partir de un muestreo por conveniencia, se conformó un grupo de 62 mujeres que cumplían con los criterios de inclusión: tener entre 25 y 69 años de edad, vivir en inmediaciones de las UI, y haber tenido una anormalidad citológica en el año previo al estudio.

\section{Procedimiento}

Las mujeres fueron contactadas telefónicamente, invitadas a participar voluntariamente y firmaron el consentimiento informado. Se realizaron ocho grupos focales, distribuidos en las cuatro uı según la edad de las participantes: cuatro grupos con mujeres de 25 a 45 años (33 participantes) y cuatro grupos con mujeres de 46 a 69 años (29 participantes). Las sesiones duraron cerca de 90 minutos, se realizaron audiograbaciones que fueron posteriormente transcritas. Como estrategia de validación, se hizo un grupo focal confirmatorio con otras seis participantes que cumplieron los criterios de inclusión y representaron los dos grupos de edad y las cuatro UI. Los resultados se presentaron al personal administrativo y asistencial de la red. El proyecto fue aprobado por el Comité de Ética de la Facultad de Salud Pública de la Universidad de Antioquia.

\section{Instrumentos}

Cuestionario sociodemográfico y guía de preguntas para explorar: los componentes del Modelo de Creencias en Salud (MCS) (18), el conocimiento acerca del $\mathrm{CaCu}$ y el proceso de atención. Las mujeres, espontánea y reiteradamente, mencionaron barreras y facilitadores del seguimiento, lo que constituyó información emergente que ameritó un análisis independiente, presentado en este artículo. Las categorías acerca de las variables psicológicas y los conocimientos se presentan en otra publicación. 


\section{Análisis}

De forma independiente, dos investigadoras realizaron un análisis de contenido $(19,20)$ y codificaron las transcripciones identificando categorías emergentes, las cuales fueron discutidas con una tercera investigadora hasta lograr consenso. Las categorías identificadas fueron discutidas en un grupo focal confirmatorio. El criterio para considerar que una categoría emergía fue la saturación: cuando las participantes hicieron alusión reiterada a contenidos similares que permitieron configurar un eje de análisis sobre las barreras o facilitadores experimentados en la búsqueda del seguimiento de alguna anormalidad citológica.

\section{Resultados}

\section{Información sociodemográfica}

Las participantes tenían entre 25 y 69 años, el 56,5\% estaban casadas o en unión libre y su nivel educativo era bajo; solo algunas terminaron bachillerato $(19,4 \%)$ y universidad $(1,6 \%)$, mientras que la mayoría únicamente tenía estudios de primaria incompleta $(35,5 \%)$, primaria completa $(24,2 \%)$, bachillerato incompleto $(16,1 \%)$ e incluso ningún estudio (3,2\%). El 67,7\% no tenía trabajo remunerado. En cuanto a la anormalidad citológica, el $88,7 \%$ requirió seguimiento y el $61,3 \%$ tratamiento; el mismo porcentaje de mujeres cuya atención dependió de alguna autorización (61,3\%). Las participantes debieron desplazarse en promedio tres veces, con el fin de tramitar autorizaciones de procedimientos o citas.

\section{Barreras del sistema de salud} grupos de edad, ni entre quienes estaban o no afiliadas al sistema de seguridad social. Los resultados se presentan en conjunto y según saturación.

Barreras estructurales. Relacionadas con la organización del sGsss y que limitaron la accesibilidad al seguimiento de las anormalidades citológicas por parte de las participantes.

\section{Fragmentación en la prestación del servicio.}

Es la principal barrera para el seguimiento de anormalidades citológicas y posibilita el avance de la enfermedad. Las mujeres tuvieron que realizar múltiples visitas a distintos y lejanos puntos de atención para hacer trámites de autorizaciones y para recibir atención. Muchas veces los trámites resultaron infructuosos y fueron remitidas a otro punto de atención sin haber recibido la autorización del procedimiento o la cita con el especialista, lo que ocasionó demora en la atención y falta de continuidad en el tratamiento.

Que ya se va a morir uno de eso, porque imagínese que a uno le dicen Sisben [a través del cual se define elegibilidad al régimen subsidiado] y cáncer, entonces muerte segura, porque de aquí a que le atiendan a uno las cosas, ya las cosas están avanzadas. (Mujer de 46 a 69 años)

Yo vine aquí [ur 1] por la orden para el examen con el ginecólogo y de allá me dijeron vaya a [ur 2] y lleva esta orden y allá se la firman y ya a ver a donde le toca. Llevé la orden allá y me dijo: no es que eso no es aquí, esto es en [Ui 3], me fui pa [u 3] y me dijeron: señora que pena esto le pertenece en [UI 4], ese mismo día me fui a pie pa [ur 4], porque no tenía con qué. Fui a [Ur 4] y me dijeron señora esto le pertenece en [UI 2], entonces al otro día volví a [u 2] y le dije mire señorita que pena yo viene con este problema ayer, esta orden y de acá me mandaron para la [Ur 3], de [Ur 3] me mandaron para [UI 4] y 
de [UI 4] me devolvieron para acá y yo traje el papelito firmado que me haga el favor y me atiendan acá y ahí si me recibieron, ahí sí. (Mujer de 46 a 69 años)

\section{Problemas con afiliaciones y características} del régimen de afiliación. Las participantes expresaron dificultades para cambiar de aseguradora o para afiliarse al régimen subsidiado luego de estar cesantes laboralmente, lo que ocasionó periodos de desafiliación sin acceso a los servicios de salud. Al perder la continuidad en la atención, algunas debieron reiniciar el proceso con la tamización:

Me sacaron del sistema de un momento a otro, yo no tengo una entidad que me respalde $[. .$.$] y todo lo que me vayan hacer$ yo lo tengo que pagar, todo. (Mujer de 25 a 45 años)

Al perder la continuidad en la atención, algunas debieron reiniciar el proceso con la tamización:

Entonces mientras hice las otras vueltas ya había pasado 2 meses [...] pa poder volver a empezar tuve que volverme a hacer la citología [...] la enfermera me dijo empecemos otra vez por la citología. (Mujer de 46 a 69 años)

Reportaron asimismo negación de la atención por parte de las aseguradoras, debido a: problemas de afiliación, falta de contratos con las instituciones que prestan los servicios y diferencias en el acceso a los procedimientos según el tipo de afiliación:

Cuando me iban a operar [...] al esposo mío se le dio por ir a manejar una finca [...] inmediatamente me metió al tal seguro, y cuando me llamaron a mí para la operación, yo resulte con que ya tenía otra salud, entonces me rechazaron esa operación. (Mujer de 25 a 45 años)
Algunas mujeres buscaron apoyo en la Personería y la Defensoría del Pueblo, instancias que brindan orientación a la ciudadanía, e iniciaron procesos para reclamar el cumplimiento de su derecho a la salud:

Me vinieron a operar apenas el 31 de Mayo. Porque me tocó poner tutela y todo. (Mujer de 25 a 45 años)

Barreras administrativas. Limitantes de la oportunidad del seguimiento, relacionadas con la prestación del servicio por parte de las aseguradoras y por la red pública hospitalaria que presta los servicios de salud.

\section{Problemas y demoras en la asignación de las} citas. Barreras en el proceso de asignación de citas: atención telefónica insuficiente, falta de personal para la remisión a especialistas, procedimientos supeditados a autorización de exámenes diagnósticos como biopsia, colposcopia y ecografía, asignación de citas para tratamiento y seguimiento de los procedimientos realizados. El retraso en la atención ocasionó, en muchos casos, el vencimiento de las órdenes médicas y meses de espera para recibir atención:

\footnotetext{
Yo me cansé, llamé, ay no, no, que no habían citas que tenía que esperar, vuelva a llamar, y yo estuve como 2 meses llamando. (Mujer de 46 a 69 años)
}

Largas filas y llegar muy temprano. Las participantes tuvieron que madrugar y hacer filas extensas para pedir citas médicas y obtener autorizaciones. El número de turnos de consulta era limitado y en varias ocasiones no fueron atendidas:

Para que a uno le toque de primeras le toca irse a uno por ahí a las 4 de la mañana, pero o sea la fila es enorme. (Mujer de 25 a 45 años) 
Problemas con autorizaciones. Las aseguradoras demoraron o negaron la autorización de servicios: exámenes diagnósticos, citas con especialistas y tratamientos; incluso los autorizados en la legislación colombiana:

Yo tuve problema con [aseguradora], porque se demoró mucho para el examen, para darme la orden pa la biopsia y nunca me la dio, entonces yo desistí. (Mujer de 25 a 45 años)

Reportaron trabas administrativas: múltiples trámites y papeleos, constantes cambios en los procesos administrativos y requerimiento de formatos especiales para solicitar el servicio, lo que les impidió agilizar el proceso y generó meses de espera sin atención médica:

Pero con esta tramitología a uno le dicen tiene un problema en la matriz, y tiene que apurarse, pilas con eso y uno va a una parte y a otra. Entonces uno piensa, el día que vaya donde el médico ya está completamente invadida y ya me voy a morir. (Mujer de 46 a 69 años)

Maltrato. Las participantes mencionaron varias experiencias de maltrato de parte del personal administrativo de las distintas instancias del sistema a las que tuvieron que acudir. La oportunidad en la atención se vio afectada por el trato agresivo de parte del personal administrativo, cuando se le pidió explicación sobre los procesos de autorizaciones; en varias ocasiones las solicitudes de las participantes no recibieron atención:

Y yo le dije, es que usted no me está atendiendo bien, es que yo no le estoy pidiendo un favor a usted, yo no le estoy pidiendo ningún favor. (Mujer de 25 a 45 años)

Barreras económicas. Las barreras estructurales y administrativas que limitaron la accesibilidad y la oportunidad del seguimiento tuvieron consecuencias económicas para las mujeres. Esta categoría fue notoria, las participantes señalaron reiteradamente que tuvieron que destinar dinero para pagar pasajes, citologías, otros exámenes diagnósticos, consultas con médicos generales y especialistas, medicamentos, tratamientos y copagos. Incluso, varias mujeres tuvieron que dejar su trabajo para destinar tiempo a tramitar autorizaciones, lo que acentuó su vulnerabilidad económica:

La plata de los medicamentos, a veces uno no tiene para pagarse una fórmula, no se la entregan, una persona pobre tiene que limitarse a quedarse sin tomar la droga porque no tenemos con qué costearla. (Mujer de 46 a 69 años)

\section{Facilitadores del sistema de salud}

Las participantes mencionaron algunas experiencias y sugerencias acerca de facilitadores que podrían mejorar la accesibilidad y la oportunidad del seguimiento de las anormalidades citológicas.

Facilitadores estructurales. Relacionados con la organización del sistema.

Evitar la fragmentación. Las mujeres mencionaron que si se dispusieran todos los trámites y la atención médica en un solo lugar y cerca al sitio de residencia, se evitaría el exceso de trámites y se lograría un mejor seguimiento de la anormalidad citológica:

Acá debería de tener un ginecólogo que lo vea a uno más frecuentemente y no tenerse uno que desplazar. (Mujer de 25 a 45 años)

Cubrir medicamentos y exámenes. Frente a los gastos de bolsillo, las mujeres consideraron necesario que haya un cubrimiento total de los gastos económicos asociados a exámenes diagnósticos, tratamiento y medi- 
camentos, los cuales deben estar siempre disponibles en las farmacias de las aseguradoras para evitar el vencimiento de las fórmulas:

Vea yo digo que la [aseguradora] lo que debería de mejorar es que le cubran a uno el tratamiento. Porque uno muchas veces por ejemplo va uno que le mandaron tantas inyecciones, tantas pastillas, pero que las inyecciones se las dan y las pastillas no las cubre. (Mujer de 25 a 45 años)

Facilitadores administrativos. Que mejoraron la prestación del servicio por parte de las aseguradoras y la red pública.

\section{Experiencias positivas en los procesos admi-} nistrativos. Contribuyeron a que la atención fuera integral y oportuna:

\begin{abstract}
Siempre me dicen, dentro de tantos días la estamos llamando y cuando me llaman, ya me tienen la cita con el ginecólogo, a tal hora, en el consultorio. (Mujer de 25 a 45 años)
\end{abstract}

Educación. Mencionaron la importancia de implementar campañas que incluyan a los hombres y que a ellas se les eduque sobre la enfermedad con charlas, campañas y difusión de información en medios de comunicación:

\begin{abstract}
Hacían campañas en los barrios de casa en casa por ejemplo la citología yo me la hice por eso [...] ¿por qué me motive a hacérmela? por eso, porque llegaba un grupo de enfermeras, vacunando, haciendo este tipo de campañas, comentando sobre el problema. (Mujer de 25 a 45 años)
\end{abstract}

Acompañamiento. Que alguien las oriente acerca del diagnóstico y el tratamiento. Las charlas y el acompañamiento de un miembro de la institución de salud podrían facilitar el tránsito por las instancias administrativas:
Una trabajadora social para que le colabore a que le digan qué vueltas tiene que hacer, o no solo las vueltas sino saber en dónde.

(Mujer de 46 a 69 años)

Mejorar la calidad del servicio. El seguimiento a las anormalidades citológicas debe ser prioritario y se debe mejorar la calidad tanto de la atención del personal que las atiende, como del servicio de salud en general:

Supongamos uno que está bien deprimido que le digan, vea usted tiene esta enfermedad, se necesita esto y esto, bueno, y entonces ya uno empieza con esos nervios, todo preocupado y que llegue allá donde le toca ir y que lo atienden a uno mal, eso lo pone a uno súper mal. (Mujer de 46 a 69 años)

Experiencias positivas en la atención. La buena atención del personal de salud y del personal administrativo facilita el proceso:

\section{Y la doctora sin embargo ella me atiende muy bien, la que me hizo la citología, ella me mantiene informada en qué me puede colaborar. (Mujer de 46 a 69 años)}

\section{Discusión}

El abordaje del cáncer de cuello uterino es una situación compleja, que amerita un estudio cuidadoso de los múltiples factores implicados en la prevención efectiva de la enfermedad. En este estudio, específicamente, se identificaron las barreras y facilitadores que experimentó un grupo de mujeres habitantes de la ciudad de Medellín y que se relacionaron con la accesibilidad y la oportunidad en la búsqueda de seguimiento de alguna anormalidad citológica. Aunque se destaca la cobertura con el tamizaje como evidencia del éxito de las acciones emprendidas para la prevención del $\mathrm{CaCu}$, 
se ha encontrado que la toma de citología se concentra en ciertos grupos, de manera que hay mujeres que no acceden a la prueba (21), ni al seguimiento en caso de anormalidad $(10,12)$. La atención integral del cáncer de cuello uterino no se reduce a la ampliación de la cobertura de la citología vaginal, la efectividad también se relaciona con la calidad de la prueba $(8,22)$ y con el seguimiento, el cual tiene mayor efecto en la reducción de la mortalidad que las coberturas elevadas de tamización $(9,23)$.

Precisamente en lo que respecta al seguimiento, en este estudio se identificaron barreras para la accesibilidad y la oportunidad que ocasionaron demoras en la atención a la mujeres y permitieron el avance de la enfermedad, lo que constituye una vulneración del derecho a la salud que, como se reportó en otros estudios, se relaciona con la organización del sGsss (24).

Las barreras de la accesibilidad al seguimiento fueron la fragmentación de la atención y los problemas de afiliación al sistema de salud. La fragmentación ha sido mencionada como un problema estructural del sistema de salud (25) y está estrechamente relacionada con las formas de afiliación, ya que la falta de integralidad en la atención surge en parte por la segmentación del sistema de salud colombiano (régimen subsidiado y contributivo) y por el modelo de competencia que lo regula, en donde las aseguradoras mediante la contratación orientada a la reducción de costos, deciden los sitios de atención sin tener en cuenta las posibles barreras geográficas y/o económicas que tendrían sus usuarias para acceder a estos sitios (26).

La fragmentación se expresó en todo el proceso de atención, en especial cuando las mujeres debían consultar en múltiples ocasiones y lugares para acceder al seguimiento. En un estudio nacional se observó que los usuarios de los servicios de salud perciben que el acceso es limitado por barreras administrativas, geográficas, normativas, de la escasa oferta y culturales, lo que genera consecuencias tan graves como un aumento en las acciones de tutela, limitaciones en la prescripción del profesional de salud, insatisfacción laboral del personal de salud, aumento en los costos de bolsillo, pérdida en la calidad de la atención y abandono de los tratamientos (16).

Se evidenció que en el momento del estudio, las actividades de primero y segundo nivel de atención, donde se encuentran el tamizaje, el diagnóstico y en algunos casos el tratamiento, estaban contratadas con diferentes instituciones prestadoras de servicios; por lo tanto, era casi imposible para el personal de la red pública, encargado solo del tamizaje, realizar un acompañamiento a la mujeres para facilitar la atención efectiva. Cabe anotar que esta problemática no es exclusiva de las acciones relacionadas con la prevención del $\mathrm{CaCu}$ y que ha sido ampliamente reportada por otros autores en referencia a otros problemas de salud (25-27).

Asimismo, es evidente la necesidad de las aseguradoras e instituciones prestadoras de servicios de implementar sistemas de información que permitan tener en red la historia clínica de las pacientes y de esta forma realizar con mayor facilidad el seguimiento cuando ellas cambian de lugar de atención o especialistas. Actualmente, la red pública hospitalaria donde se realizó la investigación realiza esfuerzos para implementar este sistema. Sin embargo, por ser una entidad pública, los recursos son limitados y puede tomar un largo tiempo la implementación de dicha estrategia.

Por otro lado, las participantes reportaron que la oportunidad de la atención se vio limitada por la demora en la asignación de citas, las largas filas que deben realizar 
para ser atendidas, los problemas con las autorizaciones y el trato inadecuado del personal administrativo. Estas limitantes se derivan de la organización del sistema de salud y de los recursos destinados a la prevención del cáncer de cuello uterino (11). Las demoras en las citas se relacionan con la cantidad insuficiente de especialistas, lo que puede referirse a los escasos recursos que se destinan al diagnóstico y tratamiento de las anormalidades citológicas. Previamente se ha reportado que el tiempo necesario para obtener cita para la toma de citología vaginal, el tiempo que debe esperar la mujer en el establecimiento para ser atendida y la insuficiente oferta de horarios de consulta de acuerdo con la demanda esperada, afectan negativamente la oportunidad para el seguimiento de anormalidades citológicas (28).

Estas situaciones son expresión de la compleja burocracia que caracteriza a la organización del sistema de salud colombiano, la cual, según se ha reportado previamente en el país, limita el acceso a los servicios y retrasa la atención por medio de mecanismos de contención de costos, lo que se traduce en consecuencias negativas para la vida de las personas $(25,29)$.

Las consecuencias económicas derivadas de los problemas en la accesibilidad y en la oportunidad de la atención se reflejaron en gastos de bolsillo, los gastos en los que incurrieron las mujeres para desplazarse a los sitios de atención, cubrir los copagos de los procedimientos y suplir los costos que no fueron asumidos por el sistema de salud. La accesibilidad geográfica les implicó a las mujeres tomar varias rutas de buses para acudir a las oficinas administrativas buscando la autorización de los procedimientos $\mathrm{y}$ tratamientos requeridos. $\mathrm{Al}$ igual que en un estudio previo (28), las participantes mencionaron dificultades para conseguir el dinero requerido para solventar gastos y los pasajes para asistir a las citas, lo que constituye una barrera para el seguimiento que reportada previamente (30). Si bien el tamizaje se realizó en los centros de salud y UI cercanas a sus viviendas, los procedimientos posteriores hicieron que tuvieran que desplazarse reiteradamente al centro de la ciudad para hacer trámites. Al igual que en estudios previos $(26,31,32)$, se observó que los gastos de bolsillo se agudizaron en mujeres de escasos recursos, afiliadas al régimen subsidiado o en condición de vinculación, lo que dio cuenta de la inequidad en la atención a esta población.

A partir de sus experiencias, las participantes propusieron algunos cambios en la disposición de los servicios de salud que podrían ser implementados, mejorando la accesibilidad y la oportunidad del seguimiento. Evitar la fragmentación de la atención y cubrir los gastos de bolsillo, además del acompañamiento de las pacientes por parte de las aseguradoras y/o instituciones prestadoras de servicios a lo largo de las instancias administrativas, la mejora en la calidad del servicio y la educación sobre la enfermedad, según las participantes, son factores que podrían mejorar el acceso al seguimiento. Este fue un hallazgo importante del estudio, acerca del cual no se encontraron antecedentes en la bibliografía revisada. $\mathrm{Si}$ bien la implementación de tales mejoras puntuales en el proceso de atención sería pertinente, estas medidas resultan secundarias frente a una modificación estructural del proceso de atención sobre la base de la garantía de la atención integral a las mujeres.

Este artículo presenta el que fue el principal hallazgo del estudio, en tanto que, como ya se mencionó, la guía de preguntas de los grupos focales no incluyó cuestiones acerca de las barreras ni los facilitadores del seguimiento relativos al sistema de salud; el objetivo inicial del estudio era explorar las variables psicológicas del MCs. Al participar en los 
grupos focales, las mujeres compartieron sus experiencias y, espontáneamente, hablaron de las barreras que tuvieron que superar para recibir el seguimiento indicado y los factores que les facilitaron el tránsito exitoso por las distintas instancias del sGsss. En este estudio cualitativo, las barreras y los facilitadores de la accesibilidad y la oportunidad del seguimiento fueron categorías emergentes que mostraron la misma situación que, desde un abordaje cuantitativo, ya se había reportado previamente en un estudio local (10). A partir de lo anterior se sustenta la importancia de articular el tamizaje y el seguimiento de las anormalidades citológicas, superando la fragmentación del sistema en la prestación de los servicios de salud.

Una de las limitaciones de este estudio es que solamente se incluyeron mujeres que habitaban en estratos socioeconómicos bajos de la ciudad de Medellín y que pertenecían al régimen subsidiado o no tenían ningún tipo de afiliación a un sistema de salud. Se recomienda explorar esta problemática en mujeres de otras zonas del departamento y del país. Debe tenerse en cuenta también que por ser este un estudio de tipo cualitativo, las participantes no fueron elegidas aleatoriamente, sino que se constituyó una muestra por conveniencia, lo que limita la generalización. Sin embargo, cabe anotar que si bien el proyecto no pretende extrapolar los resultados a lo que ocurre a nivel nacional, las barreras encontradas se relacionan con la aplicación de normas de carácter nacional y de barreras previamente descritas, por lo que es posible que este sea un reflejo de lo que está sucediendo en otros lugares de la ciudad y del país.

\section{Conclusiones}

210 Este estudio evidencia cómo el diseño del sGSSS se constituye en un obstáculo para que las mujeres se realicen el seguimiento y tratamiento de anormalidades citológicas. Es de resaltar que aun cuando una gran mayoría de mujeres se encontraban afiliadas al régimen subsidiado, esto no garantizó el acceso efectivo a los servicios. Las barreras encontradas en esta investigación llevaron a algunas mujeres al abandono del proceso de seguimiento y tratamiento, situación que es expresión de la inequidad y la vulneración de su derecho a la salud y la vida. Las mujeres con lesiones precancerosas requieren una especial atención por parte del sistema de salud, el cual debe garantizar el acceso oportuno y eficiente a un tratamiento, a través del acompañamiento permanente y la búsqueda activa de mujeres con lesiones de alto grado o superior.

\section{Agradecimientos}

A la ese Metrosalud, el Fondo de Apoyo a la Investigación Docente de la Facultad de Salud Pública de la Universidad de Antioquia y a la Estrategia de Sostenibilidad 2013-2014 de la Universidad de Antioquia.

\section{Referencias bibliográficas}

1. Tsu V, Levin C. Making the Case for Cervical Cancer Prevention: What about Equity? Reproductive Health Matters. 2008; 16 (32): 104-12.

2. World Health Organization (wHO). Global Status Report on Noncommunicable Diseases, 2010 Ginebra: wHO; 2011.

3. Global Health Council. The Burden of Cancer in Developing Countries. Global Health Council Report; 2010.

4. Ferlay J, Shin HR, Bray F, Forman D, Mathers C, Parkin DM. Estimates of Worldwide Burden of Cancer in 2008: Globocan 2008. Int J Cancer. 2010; 127 (12): 2893-917.

5. Profamilia. Encuesta Nacional de Demografía y Salud 2010. Bogotá: Profamilia; 2011.

6. Chamot E, Kristensen S, Stringer J, Mwanahamuntu M. Are Treatments for Cervical Precancerous Lesions in Less-Developed Countries Safe Enough to Promote Scaling-Up of Cervical Screening Programs? A Systematic Review. BMC Women's Health. 2010; 10: 1-11. 
7. Murillo R, Almonte M, Pereira A, Ferrer E, Gamboa OA, Jerónimo J et ál. Cervical Cancer Screening Programs in Latin America and the Caribbean. Vaccine. 2008; 26 (Suppl.) 11: L37-48.

8. Murillo R, Cendales R, Wiesner C, Piñeros M, Tovar S. Efectividad de la citología cérvico-uterina para la detección temprana de cáncer de cuello uterino en el marco del sistema de salud de Colombia. Biomedica. 2009; 29 (3): 354-61.

9. Chocontá-Piraquive LA, Alvis-Guzmán N, De la Hoz-Restrepo F. How Protective is Cervical Cancer Screening against Cervical Cancer Mortality in Developing Countries? The Colombian Case. BMC Health Serv Res. 2010; 10: 270.

10. Wiesner C, Cendales R, Murillo R, Piñeros M, Tovar S. Seguimiento de mujeres con anormalidad citológica de cuello uterino, en Colombia. Rev Salud Pública (Bogotá). 2010; 12 (1): 1-13.

11. Murillo R. Control del cáncer de cuello uterino en Colombia: triunfos y desafíos de la tamización basada en la citología cérvico-uterina. Biomédica. 2008; 28 (4): 467-70.

12. Piñeros M, Gamboa O, Hernández-Suárez G, Pardo C, Bray F. Patterns and Trends in Cancer Mortality in Colombia 1984-2008. Cancer Epidemiol. 2013; 37 (3): 233-9.

13. Baena A, Almonte M, Valencia ML, Martínez S, Quintero K, Sánchez GI. Tendencias e indicadores sociales de la mortalidad por cáncer de mama y cuello uterino. Antioquia, Colombia, 2000-2007. Salud Pública Mex. 2011; 53 (6): 486-92.

14. Ministerio de la Protección Social. Decreto 1011, Bogotá; 2006.

15. Solitario R, Garbus P, Stolkiner A. Derechos, ciudadanía y participación en salud: su relación con la accesibilidad simbólica a los servicios. Anuario de Investigaciones en Psicología. Buenos Aires: UBA; 2008.

16. Vargas J, Molina G. Acceso a los servicios de salud: Sus limitaciones y consecuencias. En: Molina G, Muñoz I, Ramírez A, editores. Dilemas en las decisiones en la atención en salud. Ética, derechos y deberes constitucionales frente a la rentabilidad financiera. Bogotá: Universidad de Antioquia, Procuraduría General de la Nación, Instituto de Estudios del Ministerio Público, Colciencias, Universidad Industrial de Santander; 2009. pp. 61-76.

17. Morales C. Evaluación de la oportunidad en la atención en el servicio de urgencias en la E.S.E. Hospital San Juan de Dios de Girón, octubrediciembre de 2008. Bucaramanga: Universidad CES y Universidad Autónoma de Bucaramanga; 2009.

18. Glanz K, Rimer BK, Lewis FM. Health Behavior and Health Education: Theory, Research, and Practice, tercera edición. San Francisco: JosseyBass; 2002.

19. Krippendorff K. Content Analysis: An Introduction to its Methodology, segunda edición. Thousand Oaks, CA, Estados Unidos: Sage; 2004.

20. Stemler S. An Overview of Content Analysis. Practical Assessment, Research \& Evaluation. 2001; 7 (17).
21. Restrepo Zea J, Mejía Mejía A, Valencia Arredondo M, Tamayo Acevedo L, Salas Zapata W. Accesibilidad a la citología cervical en Medellín, Colombia en 2006. Rev Esp Salud Pública. 2007; 81 (6): 657-66.

22. Cendales R, Wiesner C, Murillo RH, Piñeros M, Tovar S, Mejía JC. La calidad de las citologías para tamización de cáncer de cuello uterino en cuatro departamentos de Colombia: un estudio de concordancia. Biomédica. 2010; 30 (1): 107-15.

23. Andrés-Gamboa O, Chicaíza L, García-Molina M, Díaz J, González M, Murillo R et ál. Cost-Effectiveness of Conventional Cytology and HPV DNA Testing for Cervical Cancer Screening in Colombia. Salud Pública Mex. 2008; 50 (4): 276-85.

24. Echeverry-López E. Reforma a la salud y reconfiguración de la trayectoria de acceso a los servicios de salud desde la experiencia de los usuarios en Medellín, Colombia. Rev Gerenc Polit Salud. 2011; 10 (20): 97-109.

25. Abadía CE, Oviedo DG. Bureaucratic Itineraries in Colombia. A Theoretical and Methodological Tool to Assess Managed-Care Health Care Systems. Soc Sci Med. 2009; 68 (6): 1153-60.

26. Vargas I, Vázquez ML, Mogollón-Pérez AS, Unger JP. Barriers of Access to Care in a Managed Competition Model: Lessons from Colombia. BMC Health Serv Res. 2010; 10: 297.

27. Arbeláez MP, Gaviria MB, Franco A, Restrepo R, Hincapié D, Blas E. Tuberculosis Control and Managed Competition in Colombia. Int J Health Plann Manage. 2004; 19 (Suppl 1): S25-43.

28. Agüero L. Análisis de la efectividad, oportunidad, accesibilidad y satisfacción de la usuaria en el Programa de Detección Temprana del Cáncer de Cérvix según el Sistema Público de Salud y los servicios brindados por terceros en el I nivel de atención, enero a junio del 2006. San José: Instituto Centroamericano de Administración Pública (ICAP); 2007.

29. Gañán J. Los muertos de Ley 100: prevalencia de la libertad económica sobre el derecho fundamental a la salud una razón de su ineficacia caso del plan obligatorio de salud del régimen contributivo. Medellín: Universidad de Antioquia; 2013.

30. Páez A, Mercado RG, Farber S, Morency C, Roorda M. Accessibility to Health Care Facilities in Montreal Island: An Application of Relative Accessibility Indicators from the Perspective of Senior and Non-Senior Residents. Int J Health Geogr. 2010; 9: 52.

31. De Charry L, Roca S, Carrasquilla G. Usar biopsia para diagnóstico del cáncer de seno: ¿Un problema de equidad? Colomb Med. 2008; 39 (1): 24-32.

32. Mejía-Mejía A, Sánchez-Gandur AF, TamayoRamírez JC. Equidad en el acceso a servicios de salud en Antioquia, Colombia. Rev Salud Pública (Bogotá). 2007; 9 (1). 